International Journal of HRD Practice, Policy and Research 2018, Vol 3 No 1: 59-72 doi: 10.22324/ijhrdppr.3.104

\title{
Conversations About Change: Leading and Managing Change at CaseX
}

\author{
Bob Morton, ODHRM Consultants Ltd \\ Rick Holden, Liverpool Business School ${ }^{1}$
}

\begin{abstract}
That organizational change is problematic and challenging is generally acknowledged. Yet there remains a dearth of real, industry, and organizationally based case studies addressing problems, pathways, and practice experience. This article provides a reflective account of practice in relation to the leadership and management of organizational change within one part of a global chemical manufacturing company. The context in which a major programme of change was acknowledged as necessary is explained. The article then focuses on the culture change aspects, identified as critical if a range of structural, technical, and process changes were to be achieved successfully. Drawing on the experiences of senior managers involved in the change process, together with a consultant engaged as an external change agent, the article provides a rich picture discussion of key issues and themes, tensions, and questions regarding the leadership and management of the change. Conversations about change were central to the programme of culture change, providing the basis for critical connections to be made regarding workforce involvement, values, learning, and knowledge flow. Importantly, the account presented reveals the significance of the leadership team and a scenario which over time saw the emergence of a more collaborative approach to the management of the change process.
\end{abstract}

Key Words: culture change, workforce involvement, shared leadership, chemical sector

\section{Introduction}

This article offers a reflective account of practice in relation to the leadership and management of organizational change. Specifically, it focuses upon a change management journey taken by one UK plant within a multi-national chemical manufacturing company. Respecting concerns over confidentiality we cannot reveal the exact name of the particular plant nor its parent company and so will simply refer to it as 'CaseX' throughout this paper.

The article is in three parts. The first seeks to explain, in outline terms, the culture change journey taken by CaseX between 2013 and 2016. Part 2 draws on more reflective considerations and seeks to move the account from a record of the journey to a 'rich' picture discussion of key issues and themes, tensions and questions regarding the change. The third part presents an 'Editorial Discussion' which seeks to draw a summative interpretation of this account of organizational change. The paper is sourced from discussions with three members of the senior leadership team formed to drive the change at CaseX, the consultant involved as 'external change agent' with the change project from 2014 onwards, and relevant company documentation produced during this period. The paper reflects a joint enterprise between those involved in the discussions together with the Journal Editor. 


\section{Part 1: Background and Overview}

\section{Background}

CaseX's parent company operates in over 80 countries worldwide. CaseX is a large well established plant in the UK but one with a recent history of takeover and acquisition. When acquired by its current owner in 2009 it was a site with a workforce of approximately 950. The plant is engaged in the manufacture of products used to enhance industrial processing in various industries.

Whilst CaseX's corporate head office (located in mainland Europe) acknowledged that the overall performance of the plant was poor and had been starved of investment, it recognized its potential and the site fitted in with its overall organizational strategy. Senior management at the corporate head office recognized that major change was needed. However, whilst investment and restructuring were deemed necessary, there was a level of uncertainty as to the detailed nature of the plant's problems and thus no clear basis from which to develop a plan on how best to address the problems.

A consultant led review of operations, led by corporate head office, had effectively identified the main technical and process needs for the site to be profitable. The question was the leadership and implementation of the plans. Senior management within the UK recognized that, in addition to these technical and process needs, and the significant investment plans, a different approach to implementation was required. The appointment of a new site director (SD) in 2013, recruited from another company site in the UK (also an acquisition in 2009), was both symbolically and strategically significant. It was an acknowledgement from the most senior levels of the company that change at CaseX required leading from someone experienced in the sort of site reflected in the case study plant. Strategically, the new SD established certain 'modus operandi' principles in negotiation with the corporate head office. The history and the current characteristics of the site had to be acknowledged and taken on board. Critically the SD and the leadership at CaseX would be given autonomy. Change would not be imposed via a consultant led, corporate approach to the organizational change needed. The SD would determine the most appropriate leadership team to operate over coming years and any change programme would be internally driven. CaseX would be the 'masters of its own destiny'.

In the first quarter of 2013, a three-year operational improvement programme (and based on the technical review of operation noted above) was announced. This signalled the commencement of significant change at the site. The improvement programme's objectives were to achieve a transformation of the site to become a lean and reliable supplier and successful cost leader (constantly improving the cost structure to keep ahead of competition) with the agility to respond to customer and market needs. A significant capital investment programme was earmarked for the site to replace outdated equipment, introduce new technologies, change operational processes, and make the site safer. The introduction of 365 day working combined with a significant headcount reduction was a further key element of the programme's objectives and aspirations.

\section{Culture change}

The weeks and months following the announcement of the operational improvement programme were an intense period of 'conversations' about change. The SD formed a small leadership group ('think tank') to brainstorm the way forward. This included the improvement programme 
manager (also an émigré from another UK plant), the plant's HR manager and a consultant who had worked extensively with the two senior managers in the years preceding the acquisition by the CaseX parent company. Whilst organization culture had featured — almost as an afterthought - in the original head office led review, it quickly became central to the leadership group's thinking. Culture change was where the change programme needed to start ... and not with the somewhat simplistic 'guide to culture change' evident in the consultancy review, but with a much more nuanced, sensitive and intellectually informed approach to such practice.

The transformation of CaseX needed to be sustainable and be achieved in a way that equipped the people with agility to quickly adapt and cope with future changes. Critically a shift to a high-performance culture was required; a culture where the workforce as a whole performed as a well-disciplined team, where everyone was accountable and taking ownership to deliver results against shared goals. The approach needed to be one of planned involvement to gain commitment and engagement in the change process and to embed the skills of change. The change process commenced with a diagnostic phase to develop situational awareness for the change programme and subsequent analysis enabled the design of the change strategy.

\section{Achievements, 2016}

An internal document produced in 2016 asked "How do we know that change is happening?" It acknowledged that culture change is a complex and longitudinal process and change takes place over time but summed up progress to date as follows:

Most of the time, you can feel change and see it: anecdotal evidence shows that our people are behaving differently, you hear different language being used every day, stakeholders and customers are commenting on the changes they are observing. The level of participation in projects is increasing and there is active involvement and contributions to workshops and local initiatives to improve work methods and effectiveness (CaseX, 2016).

This assessment was supported by a range of quantitative and qualitative evidence, for example:

- The successful conclusion of a complex consultation on headcount reduction, job changes, working arrangements, and terms and conditions.

- The increase in the Guiding Coalition (see below) from its initial size of under 30 employees to a group of over 350 drawn from all levels of the organization.

- People who were "problem people" in the old organization are becoming stars and flourishing in the new organization where they enjoy the engagement, participation, accountability, and their voice getting heard.

- Performance data Indicating:

- Production capacity increases by over $10 \%$ at the same time as the site headcount reduces by over $20 \%$.

- $\quad 30 \%$ reduction in samples tested as a result of Quality Control and Production working together at a local level to identify process and organizational improvements.

- Customer complaints decreased from 105 in Q1, 2015 to 55 in Q4 of the same year. 
- Transfer of 25 ideas from a series of collaborative workshops, and with an estimated value of over 50 million Euros, into the strategic plans for 2016, 2017 and beyond.

Change at the site has commanded attention elsewhere within CaseX's parent company. The plant has been presented with two corporate awards; one from the national trade association and one from CaseX itself. In addition, the HR Manager presented an evidence based 'organizational change' story to one of the European corporate conferences.

This summative record of the change journey to date provides the basis for a more rigorous consideration of some of the key features of the change process, in terms of the wider debate about the difficulties and challenges facing any organization seeking to engage in the practice of organizational change.

\section{Part 2: Reflections on a Change Journey}

The practice reflection which follows is organized, initially, in respect of three key themes: problematizing; leadership development; and making connections ... unleashing potential. It is followed by a concluding discussion focused principally upon a notion of shared leadership. This latter section enables a perspective to be drawn on the theory - practice dynamic operating throughout the change process.

\section{Problematizing: context is 'king'}

Initial discussions and dialogue amongst the small leadership group reflected an iterative process of diagnosis, futures thinking and theory-practice challenges. The latter saw the group engage "intellectually" with their master's level knowledge and understanding of classic models of change (e.g. Burke \& Litwin, 1992; Kotter, 1996). Such models were important in feeding the dialogue rather than any sense that they could offer a prescriptive pathway for CaseX. Indeed, the significance of the Burke-Litwin model, for example, was most pronounced in steering the group as regards the importance of context. The crucial question was "what will work here?" There were no obvious neat solutions which could be transported from elsewhere. The solutions had to come from within. Importantly, though, there was a need to move away from assumptions, preconceptions, and generalizations about the problems.

There was insufficient communication to the site about financial performance:

[at] a fundamental level ... communication of, communication to and around the site from both leadership on the site and from leadership off the site was almost non-existent (Senior Leadership Team (SLT)).

and a need to generate a more detailed evidence base of what was (or was not happening). Whilst CaseX's corporate head office supplied a stream of financial data on the performance of the site there was a need to understand the current situation in terms of cultural and behavioural characteristics. There was a sense within the leadership group that morale, attitudes and behaviours were all problematic. To understand the context more accurately a series of explorative discussions together with a specific culture diagnostic exercise were undertaken. So, for example: 
we found out through time that we had 430 customer complaints every year on the site, so that's like more than one a day. So, that's only the customers that could be bothered complaining. So, with 430 customer complaints ... you can't expect the operator to know about every 430 customer complaints. But you would expect senior managers to have an idea about what these customer complaints are about. We didn't have any idea about what these customer complaints meant. They were all dealt with in a process but actually the process, the driver for the process was to get the complaint closed out within 28 days not to actually get to the root cause of the problem and understand what it is, what it is that needs to be changed.

When we asked, so when we asked, how often do you brief your teams, I think the last time they'd done it, we'd done one in 2012 and yeah, we did that because there was a particular reason for that and we did one in 2011, so how do you communicate it? Didn't think it was necessary ... people in key positions were not listening (SLT).

The culture diagnostic instruments (a combination of diagnostic tools drawn from Deal and Kennedy (2000) and Cummins and Worley (2004)) revealed a pervasiveness of behavioural problems. Whilst the workforce was highly dutiful, respectful and deferential, employees were overly concerned about making mistakes with a fear of being criticized/embarrassed. Overall the workforce were unwilling to take chances and/or express controversial opinions. Employees were working hard but not smartly. High performance was mistaken widely throughout the site for 'being busy':

... the focus was right across the board ... from operators who were basically coming in and just would expect to do what they were told, to shift managers who really weren't given the authority to manage and in fact we had a situation across the site where shift managers weren't aligned with their teams so you didn't have the same shift manager every shift because of the different shift patterns. So, there's no natural connection there ... And there was no expectation from the guys on the shop floor that, if you see something happening in terms of the process which you are working with every day, if you see something that's a bit different, then that might be useful information that would tell us we've got a quality problem (SLT).

A process of problematizing against this emerging contextual clarity enabled a number of key principles to be constructed by the group and which formed the basis of a blueprint for a change plan. Any transition to a future state needed to be collaborative and engaging. It needed to recognize the potential impact of the psychological impact of change. Thus, it needed to give voice to the employee and promote key values such as fairness and honesty throughout all levels of the workforce. A senior leadership team needed itself to embrace such values (and see also below). Two key 'organizing' principles in this context were structural change within the senior leadership team and the initiation of a Guiding Coalition. The former saw a proposal to reduce the leadership team from 14 to 8 as part of an initial radical re-structuring of operations. The Guiding Coalition, drew directly on Kotter's (1995) model of change management as a way of both signalling a distinctive approach to the change programme and providing a potentially catalytic vehicle to move things forward.

With the establishment of the Guiding Coalition it might be tempting to suggest that this marked the end of a problematizing process. It is probably more realistic to say that it marked the end of the beginning! The Guiding Coalition, from its inception, was involved in a continuation of the diagnostic discussions and the early mapping of a direction of travel for the change journey. The Guiding Coalition created a unified Mission and Values statement describing the desired future state for the plant, post operational improvement programme, that recognized constraints and which endeavoured to capture the new culture and degree of transformation required to be 
a successful cost leader in 2016 and beyond. It also began work on conducting a high-level gap analysis of present vs future state for each work group, its working practices and processes, the changes required and the scale of such changes.

There is a danger in any attempt to explain and outline the outcomes of the problematizing associated within CaseX in 2013/14 that a smooth and linear chronology appears to characterize its outcomes. It is more realistic to depict this a complex, inter-related, at times intense, bundle of discussions, formal and informal, producing ideas, initiatives, processes and events and all taking place simultaneously. The initiation of the Guiding Coalition and the development of its initial agenda took place the same time as the SLT was moving through its development phases. Whilst the Guiding Coalition became the principal vehicle to underpin the wider operational improvement programme and a distinctive approach to change leadership it did not displace existing, established consultation and negotiation agreements with the main trade union. Indeed, ongoing negotiations with the trade union - and underpinned with same principles noted above vis the Guiding Coalition - over a proposed shift to annualised hours, 365 days, 24 hour working (narrowly rejected in 2011), were crucial also to the success of the overall change programme and were successfully agreed in April, 2014.

Importantly the one central pillar to an otherwise complex, dynamic, and often uncertain, set of deliberations and emergent initiatives was that 'context is king'. Understanding the problems faced by the plant produced the proposed strategy for change.

\section{Leadership development}

A number of the key principles influencing the character of the change journey have been noted above. Here we address organizational leadership. The aspiration to move away from a style of leadership which was primarily exclusive required change within the existing senior leadership team (SLT) Reform of the SLT enabled clearer responsibilities to be established; ones which facilitated focused attention on an emergent change programme. A series of clusters were created, each headed up by a member of the SLT, in both the production and support sections of the site and which were designed specifically to make a statement about breaking down existing silos and positioning the SLT in terms of their leadership of the change programme. The selection of the team was conducted using a robust, fair and challenging process involving both competency based interviews and the use of high level psychometric tools in a job matching process with the objective of not only selecting leaders who could fulfil the technical aspects of the roles, but who also possessed interpersonal leadership capability and potential and who demonstrated the behaviours and values needed to achieve change and sustain the organization. This process was fully aligned with the established, corporate CaseX Competencies and Behaviours.

A series of three one-day workshops were implemented to establish a leadership team capable of taking the necessary steps towards a more collaborative approach to the change process, and with the culture change blueprint clearly in mind (see also Figure 1 below).

The workshops, held away from the site, were designed to develop the group into a high performing team, one equipped with a collective capability to lead the culture change initiative. Led by the external change agent involved in the problematizing discussed above, together with another external colleague (a business psychologist specializing in both occupational and educational psychology). The focus was personal development - as an individual, as part of a leadership team and in relation to those they would need to work with most closely (Figure 1). 
Importantly the change agent perceived the need to develop the notion of effective leadership judgement which pushed participants beyond leadership styles.

We wanted to nurture what I call rounded leadership ... yes, to encourage a shift from directive to more collaborative decision making but with care and sensitivity to quality decision making. Clear thinking and resilience is part of this. We challenged the SLT to think about their decision making ... are your decisions good enough? ... if the building is on fire you don't have a meeting (External Change Agent).

Psychometric profiles generated pre-workshop enabling each member of the SLT to work through the workshops in relation to their own personal development needs. Through themes such as empowering teams to make decisions, criticism in teams, understanding the power of feedback and the need to be better at asking for and offering help, a platform was developed for each SLT to harness their development vis a vis their own teams and as regards members of the Guiding Coalition.

\section{The Journey to Changing Culture}
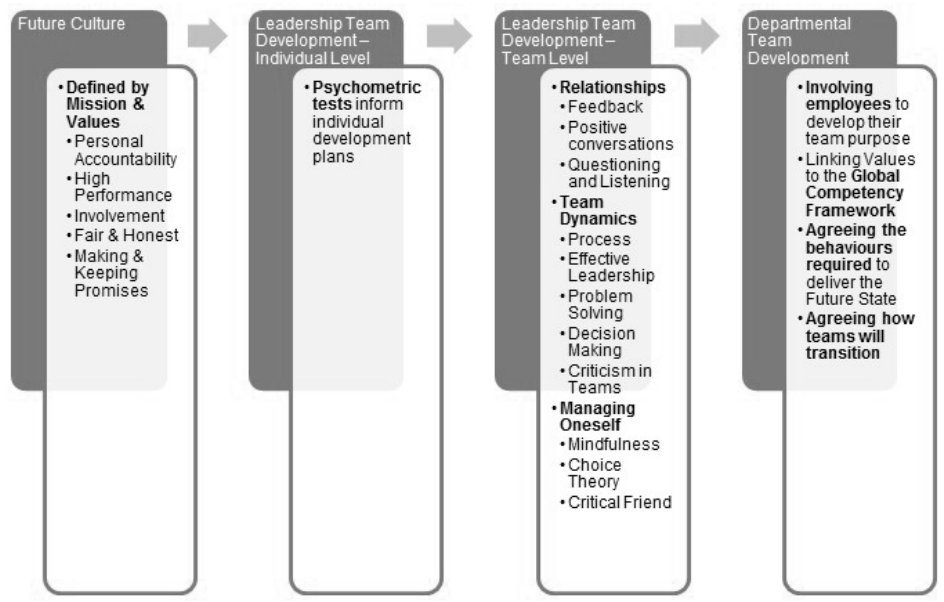

\section{Changing culture requires leadership time, commitment and persistence}

Figure 1: The Journey to Changing Culture (Source: SLT Teambuilding Workshop)

In reality there was no quick 'personal development' fix. A second workshop exposed a lack of significant progress. There remained ambiguity around an understanding of what high performance meant for the SLT themselves. Individual development work was patchy. Stronger personal commitments to areas needing developing were needed. The notion of a critical friend (Costa \& Kallick, 1993) was introduced. A critical friend is someone who is encouraging and supportive, but who also provides honest and often candid feedback that may be uncomfortable or difficult to hear (Glasser, 1998). In short, a critical friend is someone who agrees to speak 
truthfully, but constructively, about weaknesses, problems, and emotionally charged issues. Members of the SLT were 'tutored' in the skills of engagement in such processes with the help of the external change agent and which ensured such a process could be devolved to the various teams led by SLT members. By the end of the third workshop the external change agent considered sufficient progress was being made for the SLT — in relation to their teams (and in respect of the Guiding Coalition) - to discuss the behaviours required to deliver the future state. Assumptions about positional authority and control had been effectively challenged. Inclinations to be more controlling had been changed into opportunities to do things differently; to let go, reduce dependency and empower team members. For example, in relation to the members of the Guiding Coalition taking on board responsibility to present to and lead change workshops with the wider workforce see also Making connections ... unleashing potential, below.

they might not always be the best presenters, they might not always have the best slides, but actually it's much more meaningful.

There was emerging clarity about what teams throughout CaseX needed to do to make the transition and how the competences needed could be embedded as part of daily work.

\section{Making Connections ... unleashing potential}

As indicated above, there was to be no imposition of any 'packaged' or 'formulaic' solutions and importantly the proposal to utilise Kotter's notion of a Guiding Coalition helped crystallise many of the emergent principles and values surrounding a different approach to leading and managing the necessary change. The coalition needed to include individuals from all levels and areas of the organization, with the influence, energy and momentum to lead the change effort. The selection (initially totalling 35) was carried out using a set of criteria that focused on a positive attitude towards change. "We wanted people who would tell us, honestly, what they thought" (SLT).

The formation of the Guiding Coalition marked the start of a political process to change hearts and minds. It was acknowledged that mistakes would be made, that there would be uneven progress and that challenges and barriers would have to be faced. The key was to remain consistent to a set of principles encompassing involvement, listening, fairness, honesty, and consistency. In very practical terms the Guiding Coalition operated as follows. Small teams from the Guiding Coalition facilitated transition workshops, often linked to specific operational improvement programme projects e.g. re safe working and lean manufacturing. The success of these transition workshops depended upon the message and communication coming not from the SLT but from members of the Guiding Coalition with whom the workforce could identify and empathise.

getting individual members of the Guiding Coalition, not bosses, individual members to actually tell small groups of people, we described to small groups of people what transition meant for them individually, and on quite an emotional level sometimes. So, it wasn't like me or some of the other leadership team members telling people how it is, it was about individuals (SLT).

Importantly, the workshops were not about 'telling' what was to change but more of a collective conversation about work; discussions about what the participants knew best in terms of their roles, their jobs the processes, issues and problems that they were involved in on a day-day basis. 
The Guiding Coalition was the resource to make connections and help make 'conversations about change' take place. Importantly it also gave people at all levels a 'voice' which was listened to by colleagues and by management:

What we did when we thought there was something key about to happen or that we should be getting involved in, we would call together the Guiding Coalition and we pulled together workshops and then we would say, right, this is what we've done, you see what we've done, great so far, this is what we're about to do. This is the next stage. These are the things we need to be thinking about now. Tell us what, how do you think we should go about it? What's going to be our problems? What's the challenge for us? How's it, is this gonna work? You think this? And they would say, how about trying this or have you thought about that, or we can help with this, or, so it was, it became a sounding box, it's a sounding box and also a way, but it's also a way of disseminating information across the site so even people who were not on the Guiding Coalition would get news from what was happening. But we'd also use the Guiding Coalition to facilitate stuff across. If we did want to, if we did want to facilitate some workshops across the site, we would use, we would get volunteers from the guiding coalition to do that, so again it was a resource ... (SLT).

As time went by the transition workshops proved critical in a process of empowerment and shared leadership; a process through which the SLT were able to relinquish a degree of control and trust in the inherent capabilities of the work teams to deliver on operational improvement requirements.

And the best examples were from operators who suddenly made that connection that, the way our packaging machine is operating, the way we are operating our packaging machine, is causing problems that's resulting in complaints and making that link, and I can take some responsibility for that. I'm not just coming in and doing what I'm told, I'm actually going to go and actually, and with one ... we had an operator who actually went to the warehouse down the road and started telling the warehouse how they needed to slower and move our product to avoid customer complaints. Because he could see from his daily work the link between what was happening there and the number of complaints that were coming in but there was no transparency in that, no way of communicating that, no ... (SLT).

A willingness to take responsibility (cf. the key value of personal accountability (Figure 1)) and 'let go' appears to be emergent attributes:

So, it was really about trying to, um, free people up, um, and this was where the involvement came in. You know, it's like if you start getting people involved, you start giving people the freedom to do things, then actually they ... they start to use their capabilities, and, you know, we start to listen to what people are saying instead of thinking that it's the managers that know best all the time. So, whether we needed to develop capability or we needed, probably more we needed to use capabilities that we had, that we ... (SLT).

The case study, below, from one department, within CaseX captures something of how making connections through opening up communications enable the power of workforce capability to be released. 


\section{Case Study: ProceX - making connections, opening up communications, utilizing knowledge}

ProceX is one of several production processes at CaseX. It makes, using chemicals created elsewhere on site, a particular form of the major product produced on the site. With a current workforce of 58 ProceX provides an example of the early adoption of new working arrangements within the wider change programme at CaseX. Importantly, it illustrates key culture changes which enabled the wider operational improvement programme to be implemented successfully.

Senior level restructuring in 2013/4 saw the introduction of two main production clusters and as part of one of these ProceX faced a number of imperatives, including headcount reduction and the implementation of a number of early operational improvement projects. Significantly, change was required at the heart of how the plant operated; in the current shift patterns (teams not aligned with shift managers) and the non-management operating roles. Critically a shift was to be staffed with 9 operatives rather than 12, with three new roles Operative $1(\mathrm{O} 1), 2(\mathrm{O} 2)$ and $3(\mathrm{O} 3)$ (three per shift). With only a sketchy idea of the future state of the department - there was no 'manual', no predetermined new operative job descriptions - the Cluster Manager sought the engagement of the workforce from the word go. Taking the lead from the newly formed Guiding Coalition the Cluster Manager put the challenge openly and honestly to the workforce "how is this going to work?" With hindsight, this "workforce involvement" proved key to a successful transition journey lasting over 2 years. With management's encouragement, the workforce began to take a leadership role. "The guys who were doing the jobs began writing the new roles." They had the knowledge and the working experience which enabled them to fulfill this. They were able to make the connections between a future state, and which involved new equipment, with the roles that would be needed. Additionally, The ProceX workforce brought a proposal to management that they trial a nine-man shift operation prior to the site changeover to annualized hours and 24hour, 365 day working. Challenges were faced. It had to extend to more than one shift and thus, involved greater numbers. Problems were encountered, breakdowns experienced. But, issues were monitored, logged, and reflected upon by the shift teams. The learning generated was crucial in paving the way for a system that would work. This was important not just within ProceX but across the site more generally. Furthermore, it reinforced and underpinned the value of the Guiding Coalition as a key vehicle to promote and lead change. From two members on the initial Guiding Coalition many of the ProceX team became, in effect, the Guiding Coalition for the Cluster as a whole, thereby developing a momentum.

The preparedness to engage in decision making about roles, shift patterns etc. marked the beginnings of a move to a more general ability to operate in self-managed teams. This became important in three key areas:

- Workforce development: training for the new operative roles; cross-training to increasingly multiskill the workforce; much of which is now implemented in situ using experienced employees trained in job instruction.

- Day to day problem solving and operations management: demonstrating high levels of responsible decision-making.

- New ideas: low cost high value improvements to plant processes; often from a safety perspective.

The sense of ownership in the achievements of the 'non-management' ProceX workforce over two plus years, is evident in their display located at the entrance to the ProceX plant. The display is generated using the words of the workforce themselves. It is testimony to a change journey influenced (led) by the workforce rather than change being done to them. 


\section{Part 3 Editorial Discussion: Towards Shared Leadership?}

It is regularly asserted that over two-thirds or more of organizational change initiatives fail to achieve their intended aims (Hamlin, 2016). Whilst such claims lack any strong empirical foundations they serve nonetheless to highlight the challenge organizational change presents for those engaged in its leadership and management. Indeed, they cast a shroud of uncertainty over the voluminous literature produced over the last 50 years on leadership and management of organizational change to guide and influence those involved in its practice. Dominant in much of this literature is attention to leadership itself. Here the 'great man' theory of leadership continues to be hugely influential (Badaracco, 2001). It continues to underpin the appointment of chief executives, 'head-hunted', from other organizations and often charged with 'turning the organization around'. Reflection on what unfolded at CaseX reveals a much more nuanced picture. Undoubtedly a 'new man at the top' was influential in getting things moving and setting a strategic direction but the new site director was first and foremost an internal appointment; someone who 'knew the business' and who was able to avoid the tag of leadership from the corporate centre. Furthermore, this CaseX story is not one of a change programme initiated and delivered by a single charismatic individual. The account presented reveals the significance of a leadership team and a scenario which over time saw the emergence of a more collaborative approach to the delivery of the change process.

Leadership in any context has historically been described in relation to the behaviour of an individual and their relationship to their followers. This has resulted in an emphasis in leader/ leadership training on the behaviour, characteristics and actions of leaders. However, in more recent times - and in part reflecting the lack of research evidence for a single individual to have the kind of dramatic impact on organizational performance that is so often claimed (Thorpe et al., 2011) — "post heroic" ideas have emerged emphasizing the value of more collaborative and less hierarchical practices (Collinson, 2008). Thus 'shared leadership' implies an activity that is shared, or distributed, among members of the team and which characterizes its way of working. A key distinction between shared and traditional models of leadership is that the influence process involves more than just downward influence of sub-ordinates by a positional leader (Pearce, Manz \& Sims, 2009). Leadership is distributed amongst a set of individuals instead of being centralized in the hands of a single individual who acts in the role of leader (Pearce \& Conger, 2002, pp. 1-3). Each team member's individual experience, knowledge and capacity is valued and is used by the team to distribute or share the job of leadership through the team in response to each context and challenge being faced. In very practical, pragmatic terms it is captured well by the words of one CEO working in the UKs health service:

\footnotetext{
Shared leadership works on the principle that teams work together more effectively to deliver high quality patient care if all members assert their individual leadership qualities. This does not mean no-one takes ultimate control but, if the team leader has a day off or moves, that the team continues to work effectively. The days of naive reliance on a single hero who can supposedly solve everyone's problems are over (Thornton, 2007).
}

Thornton's point about control raises important questions about change and change management/ leadership at CaseX. Kotter's model of change management, and influential within this account, not least because of the Guiding Coalition, could be interpreted as a top down intervention; a set of steps driven by the site director, together with clear plans and schedules for each stage. Undoubtedly the new Site Director saw the ultimate control, as resting with himself. It was in his power to develop a new Senior Leadership Team and initiate the Guiding Coalition, embark 
on leadership development (for the senior team) and prepare plans for how culture change was to be realized to support and feed into the ongoing operational improvement programme. Whilst an overt, and theoretically driven strategy to bring about shared leadership was not evident, an attempt to engender engagement and collaboration vis the change programme was clearly part of the adopted leadership approach. Issues of control and the relationships necessary to build a different culture were central to Senior Leadership Team and Guiding Coalition deliberations:

... but it's almost the impact, the impact from the guiding coalition members almost I would say increased as you moved away from leadership team down to operators because when you get an operator, when you get an operator stand up and telling other operators or other people what they've done, it's much more meaningful (SLT).

If you're the boss and everybody does as you say and they always do as you say, you don't actually need a relationship because you just do as you're told. But actually, once you want to get more to an engaging and involved culture, and you want to move that spectrum, then you've got to have a relationship because if you don't have a relationship, you can't manage in that way (External Change Agent).

The important notion is perhaps that of 'managing in a different way' (cf. extract 2, above) and which raises the question of necessary leadership development vis 'shared leadership'. Change to bring about change. For many years change has been equated with learning (Gold et al., 2012) and clearly the CaseX story is no exception. A spectrum of formal and informal learning has underpinned the culture change and indeed underpins the emergent shared leadership. The importance of leadership development to manage in a different way is a feature of Part 2 of this article and it is useful to note here that in terms of developing leadership capabilities beyond the SLT that this is recognized and part of the Guiding Coalition's agenda to help sustain the change programme.

so what the leadership team's been through in terms of their own development and their development within a team is not yet as advanced in the rest of the organization as it should be ... and I think that's gonna take a bit of time to do that work in the rest of the organization (SLT).

In sum, therefore, whilst initially driven from the top this account of practice at CaseX, and the illustrative evidence upon which it draws, provides strong testimony to processes of shared leadership beginning to take hold and facilitate the change journey. They have helped to secure context specific culture change; change which underpinned performance improvements in line with the imperatives of the operational improvement programme and a return to profitability.

In a recent article questioning the continued adherence to traditional notions of 'training' Cole (2017) advances a practice framework integrating ideas about 'connections', 'conversations', and 'knowledge'. Cole argues that for an organization to change and develop, knowledge needs to flow freely and be pooled where it is required. Connections are critical to making an organization work well and conversation is the key way to support both the flow of knowledge and the essential connectivity of people. From the initial leadership group and newly formed SLT through to the Guiding Coalition and the dialogue at shop floor level, an integration of connections and conversations to enhance knowledge flow provides a powerful summary of how a critical level of (sustainable) shared leadership was nurtured and became impactful at CaseX. It is testimony to how 'connections', 'conversations', and 'knowledge' are at the heart of shared leadership. Conversations about change were central to a programme of culture change at CaseX; intellectual conversations about change leadership through to practical and 
pragmatic conversations about the realities of making transition journeys. A fitting conclusion is to highlight the value of conversations about talent in any change context; where talent resides within an organization; the perils of overlooking such potential in deference to I/we know best; and how through processes of shared leadership an inclusive approach to talent management can pay real dividend.

The evidence presented in this paper suggests an account of practice which has been successful. However it makes no strong claim regarding 'best practice'. The complexity and uniqueness of any organizational change, organization by organization, makes any such claim largely futile. What the paper as whole has sought to generate is insight into the sorts of conversations about the leadership and management of change which need to take place and which, appropriately harnessed, can enrich our understanding of its practice.

\section{Notes}

1 The Journal acknowledges the contribution of three members of CaseX in the authorship of this article but who have specifically requested that they remain anonymous, respecting their company policy on such matters.

\section{References}

Baradacco, J. L. (2001). We don't need another hero, Harvard Business Review, 79(8), 120-126.

Burke, W. W., \& Litwin, G. H. (1992). A Causal Model of Organisation Performance and Change, Journal of Management, 18(3), 523-545.

CaseX (2016) European Best Practice: The leadership journey to changing culture, CaseX unpublished internal report .

Cole, M. (2017). Rethinking the practice of workplace learning and development: utilizing 'knowledge, connections and conversation'; in organizations, International, Journal of HRD Practice, Policy and Research, 2(1), 7-19; DOI:10.22324/ijhrdppr.2.102

Collinson, D. (2008). Distributed and Shared Leadership CEL Practitioner Research Projects 2007-08, Volume 8, Centre for Excellence in Leadership, University of Lancaster, https://www.lancaster.ac.uk/ media/lancaster-university/content-assets/documents/lums/lsis/vol8.pdf Accessed 4 May, 2017.

Costa, A. L., \& Kallick, B. (1993). Through the lens of a critical friend, Educational leadership: journal of the Department of Supervision and Curriculum Development, N.E.A 51(2)·January 1993.

Cummings, Thomas G., \& Worley, Christopher G. (2004). Organization Development and Change, 8th Ed., South-Western College Pub.

Deal T. E., and Kennedy, A. A. (1982, 2000). Corporate Cultures: The Rites and Rituals of Corporate Life, Harmondsworth, Penguin Books, 1982; reissue Perseus Books, 2000.

Glasser, W. (1998). Choice theory: a new psychology of personal freedom. New York: Harper Perennial.

Hamlin, R. (2016). HRD and Organizational Change: Evidence Bases Practice, International Journal of HRD Practice, Policy and Research, 1(1), 7-20; DOI: 10.22324/ijhrdppr.1.102

Kotter, J. P. (1996). Leading Change. Boston: Harvard Business School Press.

Pearce, C. L., \& Conger, J. A. (2002). All Those Years Ago: The Historical Underpinnings of Shared Leadership in C. L. Pearce, and J. A. Conger (eds.) Shared Leadership: Reframing the Hows and Whys of Leadership, Thousand Oaks, CA: SAGE Publications pp. 1-18.

Pearce, C. L., Manz, C. C., and Sims Jnr., H. P. (2009). Where Do We Go From Here: Is Shared Leadership the Key to Success? Organisational Dynamics, 38(3), 234-238.

Thornton, S. (2007). Stephen Thornton on shared leadership, Health Service Journal, http://www.hsj.co.uk/ stephen-Thornton-on-shared-leadership/3248.article Accessed: 27 April, 2017. 
Thorpe, R., Gold, J., and Lawlor, J. (2011). Locating Distributed Leadership, International Journal of Management Reviews, 13(3), 239-250. DOI: 10.1111/j.1468-2370.2011.00303.x

\section{The Authors}

Bob Morton founded ODHRM Consultants Ltd in 2010. He is President of the European Association of People Management and Chair of CIPD Enterprises Ltd. Until 2009 he was Global Head of OD for Swiss based Ciba Specialty Chemicals.

Rick Holden is Research Fellow at Liverpool Business School. Until 2014 he was Principal Lecturer at Leeds Business School. He has been Editor of The International Journal of HRD Practice, Policy \& Research since its launch in 2016 and is News Editor for the European Association of People Management. 